\title{
964.
}

\section{ON THE NINE-POINTS CIRCLE OF A SPHERICAL TRIANGLE.}

[From the Quarterly Journal of Pure and Applied Mathematics, vol. xxvII. (1895), pp. 35-39.]

THE definition is in effect given in Hart's paper, "Extension of Terquem's theorem respecting the circle which bisects three sides of a triangle," Quarterly Mathematical Journal, t. IV. (1861), pp. 260, 261, viz. if we have a spherical triangle $A B C$, then we have a circle (i.e. a small circle of the sphere), say the nine-points circle, meeting the sides $B C, C A, A B$ in the points $F, L ; G, M ; H, N$ respectively, where

$$
\begin{aligned}
& \tan \frac{1}{2} B F^{\prime}=\frac{\cos \frac{1}{2} b-\cos \frac{1}{2} c \cos \frac{1}{2} a}{\cos \frac{1}{2} c \sin \frac{1}{2} a} \\
& \tan \frac{1}{2} C F^{\prime}=\frac{\cos \frac{1}{2} c-\cos \frac{1}{2} a \cos \frac{1}{2} b}{\cos \frac{1}{2} b \sin \frac{1}{2} a}
\end{aligned}
$$

(which equations agree with $B F+F C=B C$ ), and

$$
\begin{aligned}
& \tan \frac{1}{2} B L=\frac{\cos \frac{1}{2} c \sin \frac{1}{2} a}{\cos \frac{1}{2} b+\cos \frac{1}{2} c \cos \frac{1}{2} a} \\
& \tan \frac{1}{2} C L=\frac{\cos \frac{1}{2} b \sin \frac{1}{2} a}{\cos \frac{1}{2} c+\cos \frac{1}{2} a \cos \frac{1}{2} b}
\end{aligned}
$$

(which equations agree with $B L+C L=B C$ ); and with the like formulæ for the points $G, M$ : and $H, N$ : respectively. write

If, as usual, the sides of the triangle are called $a, b, c$, and for shortness we $\left(\cos \frac{1}{2} a, \cos \frac{1}{2} b, \cos \frac{1}{2} c ; \sin \frac{1}{2} a, \sin \frac{1}{2} b, \sin \frac{1}{2} c\right)=\left(p, q, r ; p_{1}, q_{1}, r_{1}\right)$, 
then the formulæ are

and

$$
\begin{aligned}
& \tan \frac{1}{2} B F=\frac{q-r p}{r p_{1}}, \quad \tan \frac{1}{2} C F=\frac{r-p q}{q p_{1}} \\
& \tan \frac{1}{2} C G=\frac{r-p q}{p q_{1}}, \quad \tan \frac{1}{2} A G=\frac{p-q r}{r q_{1}} \\
& \tan \frac{1}{2} A H=\frac{p-q r}{q r_{1}}, \quad \tan \frac{1}{2} B H=\frac{q-r p}{p r_{1}}
\end{aligned}
$$

$$
\begin{aligned}
& \tan \frac{1}{2} B L=\frac{r p_{1}}{q+r p}, \quad \tan \frac{1}{2} C L=\frac{q p_{1}}{r+p q}, \\
& \tan \frac{1}{2} C M=\frac{p q_{1}}{r+p q}, \quad \tan \frac{1}{2} A M=\frac{r q_{1}}{p+q r}, \\
& \tan \frac{1}{2} A N=\frac{q r_{1}}{p+q r}, \quad \tan \frac{1}{2} B N=\frac{p r_{1}}{q+r p} .
\end{aligned}
$$
have

Before going further, it may be remarked that for $a, b, c$, each of them small, we

$$
\frac{1}{2} B F=\frac{\left(1-\frac{1}{8} b^{2}\right)-\left(1-\frac{1}{8} c^{2}\right)\left(1-\frac{1}{8} a^{2}\right)}{\frac{1}{2} a},
$$

that is, $B F=\frac{a^{2}+c^{2}-b^{2}}{2 a}=c \cos B$, and similarly $C F=a \cos C$, if $A, B, C$ are the angles of the plane triangle; that is, in the plane triangle $F, G, H$ are the feet of the perpendiculars let fall from the angles on the opposite sides. Moreover, $\tan \frac{1}{2} B L=\frac{\frac{1}{2} a}{1+1.1}$, that is, $B L=\frac{1}{2} a$, and similarly $C L=\frac{1}{2} a$; that is, $L, M, N$ are the median points of the three sides respectively.

In the general case of the spherical triangle $A B C$, the construction is effected by means of a triangle $A^{\prime} B^{\prime} C^{\prime}$, the sides whereof are respectively the halves of those

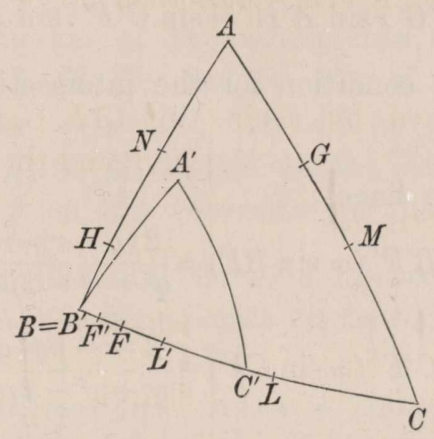

of the original triangle: viz. for this triangle $A^{\prime} B^{\prime} C^{\prime}$, we construct the points $F^{\prime}, G^{\prime}, H^{\prime}$ and $L^{\prime}, M^{\prime}, N^{\prime}$, and then on the sides of the triangle $A B C$ taking $B F^{\prime}=2 B F^{\prime \prime}$, $B L=2 B L^{\prime}, \& c$., we have the points $F, G, H, L, M, N$. 
Thus $p, q, r, p_{1}, q_{1}, r_{1}$ denoting as above the cosines and sines of the half-sides of the triangle $A B C$, that is, the cosines and sines of the sides of the triangle $A^{\prime} B^{\prime} C^{\prime}$, we have

$$
\begin{gathered}
\tan B^{\prime} F^{\prime}=\frac{q-r p}{r p_{1}}, \quad \tan C^{\prime} F^{\prime}=\frac{r-p q}{q p_{1}} \\
\vdots \\
\tan B^{\prime} L^{\prime}=\frac{r p_{1}}{q+r p}, \quad \tan C^{\prime} L^{\prime}=\frac{q p_{1}}{r+p q} \\
\quad
\end{gathered}
$$

First, for the points $F^{\prime}, G^{\prime}, H^{\prime}$, we have $A^{\prime} F^{\prime}, B^{\prime} G^{\prime}, C^{\prime} H^{\prime}$, the perpendiculars from the angles on the opposite sides, meeting in a point $O^{\prime}$, the orthocentre of the triangle $A^{\prime} B^{\prime} C^{\prime}$ : in fact, from the triangle $A^{\prime} B^{\prime} F^{\prime \prime}$, right-angled at $F^{\prime}$, we have

$$
\begin{aligned}
\tan B^{\prime} F^{\prime}=\tan A^{\prime} B^{\prime} \cos B^{\prime} & =\tan \frac{1}{2} c \frac{\cos \frac{1}{2} b-\cos \frac{1}{2} c \cos \frac{1}{2} a}{\sin \frac{1}{2} c \sin \frac{1}{2} a} \\
& =\frac{r_{1}}{r} \frac{q-r p}{r_{1} p_{1}}=\frac{q-r p}{r p_{1}}
\end{aligned}
$$

as above, and similarly for the points $G^{\prime}$ and $H^{\prime}$.

I notice that we have

and thus

$$
\sin B^{\prime} F^{\prime}=\frac{q-r p}{\sqrt{ }\left\{(q-r p)^{2}+r^{2} p_{1}^{2}\right\}}, \quad=\frac{q-r p}{\sqrt{ }\left(q^{2}+r^{2}-2 p q r\right)},
$$

hence

$$
\begin{array}{ll}
\sin B^{\prime} F^{\prime}=\frac{q-r p}{\sqrt{\left(q^{2}+r^{2}-2 p q r\right)}}, & \sin C^{\prime} F^{\prime}=\frac{r-p q}{\sqrt{ }\left(q^{2}+r^{2}-2 p q r\right)} \\
\sin C^{\prime} G^{\prime}=\frac{r-p q}{\sqrt{\left(r^{2}+p^{2}-2 p q r\right)}}, & \sin A^{\prime} G^{\prime}=\frac{p-q r}{\sqrt{\left(r^{2}+p^{2}-2 p q r\right)}} \\
\sin A^{\prime} H^{\prime}=\frac{p-q r}{\sqrt{\left(p^{2}+q^{2}-2 p q r\right)}}, & \sin B^{\prime} H^{\prime}=\frac{q-r p}{\sqrt{\left(p^{2}+q^{2}-2 p q r\right)}}
\end{array}
$$

$\sin B^{\prime} F^{\prime} \cdot \sin C^{\prime} G^{\prime} \cdot \sin A^{\prime} H^{\prime}=\sin C^{\prime} F^{\prime} \cdot \sin A^{\prime} G^{\prime} \cdot \sin B^{\prime} H^{\prime}$,

which (as is well known) is the condition for the intersection of the arcs $A^{\prime} F^{\prime}, B^{\prime} G^{\prime}, C^{\prime} H^{\prime}$ in the orthocentre $O^{\prime}$.

But I say further that we have

$$
\begin{aligned}
& \sin 2 B^{\prime} F^{\prime}\left(=\sin B F^{\prime}\right)=\frac{2(q-r p) r p_{1}}{q^{2}+r^{2}-2 p q r}, \\
& \sin 2 C^{\prime} F^{\prime}(=\sin C F)=\frac{2(r-p q) q p_{1}}{q^{2}+r^{2}-2 p q r}
\end{aligned}
$$

and thence

$$
\sin B F \cdot \sin C G \cdot \sin A H=\sin C F \cdot \sin A G \cdot \sin B H,
$$

and thus the arcs $A F, B G, C H$ meet in a point which is obviously not the orthocentre of the triangle $A B C$. 
Secondly, for the points $L^{\prime}, M^{\prime}, N^{\prime}$, we have

that is,

$$
\sin B^{\prime} L^{\prime}=\frac{r p_{1}}{\sqrt{\left(q^{2}+r^{2}+2 p q r\right)}}, \quad \sin C^{\prime} L^{\prime}=\frac{q p_{1}}{\sqrt{\left(q^{2}+r^{2}+2 p q r\right)}}
$$

and similarly

$$
\sin B^{\prime} L^{\prime}: \sin C^{\prime} L^{\prime}=r: q, \quad=\cos B^{\prime} A^{\prime}: \cos C^{\prime} A^{\prime}
$$

$$
\begin{aligned}
& \sin C^{\prime} M^{\prime}: \sin A^{\prime} M^{\prime}=p: r,=\cos C^{\prime} B^{\prime}: \cos A^{\prime} B^{\prime}, \\
& \sin A^{\prime} N^{\prime}: \sin B^{\prime} N^{\prime}=q: p,=\cos A^{\prime} C^{\prime}: \cos B^{\prime} C^{\prime}
\end{aligned}
$$

viz. the sides $B^{\prime} C^{\prime}, C^{\prime} A^{\prime}, A^{\prime} B^{\prime}$ are by the points $L^{\prime}, M^{\prime}, N^{\prime}$ divided each into two parts such that for any side the sines of the two parts are proportional to the cosines of the other two sides. We have

$$
\sin B^{\prime} L^{\prime} \cdot \sin C^{\prime} M^{\prime} \cdot \sin A^{\prime} N^{\prime}=\sin C^{\prime} L^{\prime} \cdot \sin A^{\prime} M^{\prime} \cdot \sin B^{\prime} N^{\prime},
$$

viz. the arcs $A^{\prime} L^{\prime}, B^{\prime} M^{\prime}, C^{\prime} N^{\prime}$ meet in a point $K^{\prime}$ which may be called the cos-centre of the triangle $A^{\prime} B^{\prime} C^{\prime}$ (where observe that, for $a, b, c$ indefinitely small, i.e. for a plane triangle, the points $L^{\prime}, M^{\prime}, N^{\prime}$ are the mid-points of the sides, and the centre $K^{\prime}$ is the c. G. or median point of the triangle).

But further, we have

and thence

$$
\begin{gathered}
\sin 2 B^{\prime} L^{\prime}(=\sin B L)=\frac{2 r p_{1}(q+r p)}{q^{2}+r^{2}+2 p q r} \\
\vdots \\
\sin 2 C^{\prime} L^{\prime}(=\sin C L)=\frac{2 q p_{1}(r+p q)}{q^{2}+r^{2}+2 p q r} \\
\vdots
\end{gathered}
$$

$\sin B L \cdot \sin C M \cdot \sin A N=\sin C L \cdot \sin A M \cdot \sin B N$,

viz. the arcs $A L, B M, C N$ meet in a point, which is obviously not the cos-centre of the triangle $A B C$.

We have thus the construction of the nine-points circle as a six-points circle, by means of the points $F, G, H, L, M, N$; and by way of recapitulation we may say that the nine-points circle meets the sides $B C, C A, A B$ in the points $F, L ; G, M ; H, N$ respectively, where the points $F, G, H$ depend on the ortho-centre of the semi-triangle, and the points $L, M, N$ depend on the cos-centre of the semi-triangle.

The triangle $A B C$ has an inscribed circle and three escribed circles, and we have (as is known) the theorem that the nine-points circle touches each of these four circles. The circles $B C, C A, A B$ and the nine-points circle form a tetrad of circles, and the inscribed circle and the three escribed circles a tetrad of circles, or say the eight circles form a bitetrad, such that each circle of the one tetrad touches each circle of the other tetrad. 